\title{
De-Escalation of Breast Cancer Surgery Following Neoadjuvant Systemic Therapy
}

\author{
(1) Umar Wazir ${ }^{1,2}$, (10) Kefah Mokbel ${ }^{1}$
}

${ }^{1}$ London Breast Institute, Princess Grace Hospital, London, United Kingdom

${ }^{2}$ Department of General Surgery, Khyber Teaching Hospital, Peshawar, Pakistan

\section{ABSTRACT}

Breast cancer treatment has seen many advances in recent decades, lessening the morbidity to patients, while improving outcomes. Central to these gains has been the introduction of breast conserving surgery and neoadjuvant systemic therapy (NST). There is a considerable interest in further de-escalation of the treatment of breast cancer, which is being studied in several ongoing randomised trials. We aimed to appraise the current literature regarding the various aspects of de-escalation of surgical treatment of breast cancer after NST, and attempt to prognosticate the future course of breast oncotherapy.

Keywords: Breast cancer, neo-adjuvant therapy, de-escalation

Cite this article as: Wazir U, Mokbel K. De-Escalation of Breast Cancer Surgery Following Neoadjuvant Systemic Therapy.

Eur J Breast Health 2022; 18(1): 6-12

\section{Key Points}

- De-escalation of breast cancer treatment aims to reduce morbidity and improve quality of life without compromising the oncological outcome.

- Patients with triple-negative or HER2 positive breast cancer who achieve an excellent response to NST are suitable candidates for de-escalation of breast cancer surgery.

- Patients with cN1-2 disease before NST who become cN0 after NST can undergo TAD as an alternative to complete ALND if pCR is achieved.

- The elimination of lumpectomy following an excellent response to NST remains the subject of ongoing clinical trials.

\section{Introduction}

Currently, over $20 \%$ of patients with early breast cancer are treated with neoadjuvant systemic therapy (NST) and this proportion has been increasing over the years (1). Initially, the reason for offering NST was downstaging locally advanced tumours to facilitate breast-conserving surgery (BCS). However, its role has expanded to include other aims such as in vivo drug sensitivity testing and provision of critical prognostic information that can guide and tailor adjuvant treatment for residual disease. The use of adjuvant systemic therapy in patients with residual disease following NST for triple negative breast cancer (TNBC) or human epidermal growth factor receptor 2 (HER2) positive breast cancer has been shown to improve overall survival $(2,3)$.

Furthermore, advances in NST protocols have increased the rates of observed pathological complete response (pCR). Examples of such refinements in current NST protocols include carboplatin for TNBC (4), the addition of pertuzumab to trastuzumab for HER2 positive breast cancer (5), and more recently, the addition of immunotherapy for TNBC (6).

In addition to the marked improvements in pCR rates, there is a growing body of evidence from randomised controlled trials (RCTs) that omission of surgery for minimal residual disease in the axilla outside the NST does not compromise oncological outcome. Two randomised trials have demonstrated that omission of complete axillary lymph node dissection (ALND) when the sentinel lymph node biopsy (SLNB) is positive for malignancy does not compromise the overall survival. The American College of Surgeons Oncology Group (ACSOG) Z0011 trial showed that patients with clinically node-negative breast cancer with 1-2 positive sentinel lymph nodes achieved equivalent overall (OS) and disease-free survival (DFS) compared to those undergoing ALND (7). 
In the AMAROS trial, patients with positive SLNB undergoing axillary radiation therapy had a similar OS to those undergoing ALND (8). The axillary local recurrence rate was slightly higher in the radiation group and incidence of lymphedema was higher in the ALND arm. The SLNB procedure is known to have a recognised false negative rate (FNR) in patients with cN0 disease of up to $10 \%$. However, the various RCTs comparing the SLNB and ALND in patients with a clinically node-negative breast cancer have shown equivalent oncological outcomes (9). These observations have inspired research into the potential for de-escalating breast cancer surgery following NST. In view of the limited evidence regarding surgery, de-escalation after neoadjuvant endocrine therapy, neoadjuvant chemotherapy +/immunotherapy will be the focus of this article.

\section{Predictors of pCR}

Patients with TNBC or HER2 positive disease are known to have the highest rates of pCR in the breast (10). Other predictors of high pCR include high tumour grade and high proliferation index. Hormone sensitive lobular breast cancer is known to achieve the lowest rate of pCR. Axillary pCR seems to be higher than that of the breast, although breast pCR is the best predictor of axillary pCR (11).

Patients with triple-negative or HER2 positive breast cancer who achieve CCR in the breast would be excellent candidates for deescalation of breast cancer surgery. Therefore, it is critical that imaging modalities accurately predict pCR so that de-escalation of breast cancer surgery can be accurately planned. However, it should be noted that breast cancer patients who have a partial or complete response in imaging, as well as pCR, are also candidates for de-escalation in surgery.

We have recently reviewed the evidence regarding the potential role of positron emission tomography (PET) in the assessment of axillary disease and concluded that 2-deoxy-2-[fluorine-18]fluoro- D-glucose integrated with computed tomography $\left({ }^{18} \mathrm{~F}-\mathrm{FDG} \mathrm{PET} / \mathrm{CT}\right)$ has a low sensitivity but high specificity for axillary nodal disease. Therefore, ultrasound-guided biopsy could be considered in a positive computed tomography/Positron emission tomography (CT/PET). Modest accuracy however prohibits the use of ${ }^{18} \mathrm{FDG}-\mathrm{PET} / \mathrm{CT}$ alone in axillary staging. Prospective research using standardised protocols and quantitative cut-off points is warranted (12).

Moreover, the diagnostic performance of non-invasive imaging, including PET and magnetic resonance imaging (MRI), for assessment of axillary response after NST in clinically node-positive breast cancer was the focus of a recent systematic review and meta-analysis. The authors concluded that the diagnostic performance of current noninvasive imaging modalities was too limited to accurately assess axillary response after NST in clinically node-positive breast cancer patients (13).

Breast MRI seems to be the most accurate modality for predicting pCR of the primary tumour (Figure 1) and has received the highest rating (rated 9) by the American College of Radiologists (14, 15). A recent meta-analysis has demonstrated that contrast-enhanced breast MRI has performed well in predicting pCR of the primary tumour with a pooled sensitivity of $80 \%$ and specificity $84 \%$ (16).

Ultrasonography (rated 8) represents the second-best modality for monitoring the primary tumour response in the breast and is valuable in countries with limited MRI resources. In relation to monitoring the nodal response in patients with initially node-positive breast cancer, ultrasonography seems to be the gold standard (Figure 2) (15).

\section{Clinically Node-Negative Breast Cancer (cN0)}

SLNB following NST in patients with clinically node-negative (cN0) breast cancer has been shown to be equivalent to SLNB prior to treatment. A recent meta-analysis (17) reported an identification rate of $96 \%$ and a false negative rate (FNR) of $6 \%$ in post-NST SLNB. There was no significant difference in OS or DFS, thus confirming the oncological safety of this approach $(18,19)$.

Furthermore, SLNB performed post-NST is more likely to be negative, thus down-staging axillary disease and reducing the rate of ALND. In view of the clear evidence regarding the efficacy of this approach, SLNB after NST has become the gold standard in patients with clinically node-negative breast cancer undergoing NST, as reflected by its incorporation into international guidelines (20). A prospective cancer registry study in Germany recorded that almost $100 \%$ of

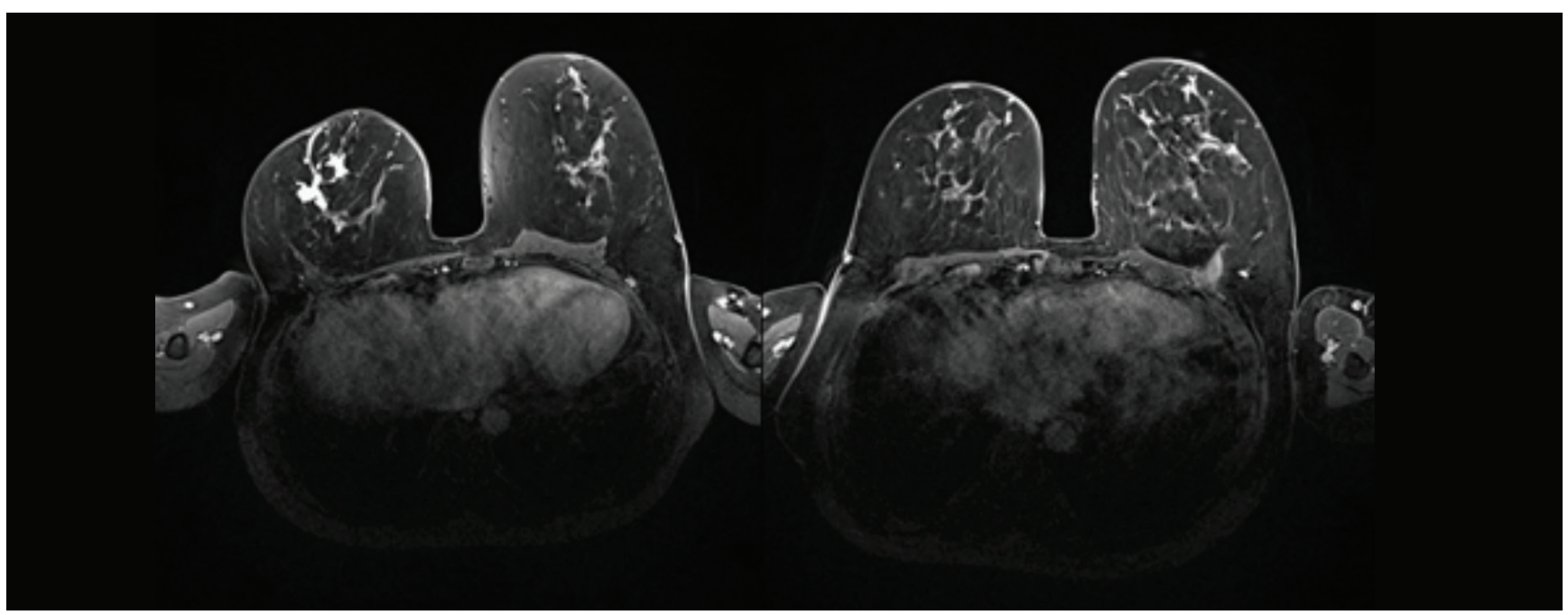

Figure 1. MRI demonstrating clinical complete response (cCR) of recurrent node positive TNBC in the right breast to NST that included Carboplatin and Pembroluzimab in a 50-year old woman (left: before NST; right: after NST. The patient achieved pCR. 
breast cancer patients underwent complete ALND 2008. However, the number of patients undergoing this radical surgical approach has declined sharply to $24.4 \%$ in 2016 (21).

Barron et al. (22) reported that in clinically node-negative, triplenegative or HER2 positive breast cancer who achieve PCR in the breast following NST the incidence of residual nodal disease was less than $2 \%$. The authors reported a nodal pCR of $98.4 \%$ in such patients when analysing data spanning more than 4,000 patients in the National Cancer Database. The incidence of positive SLNB was found to be $1.6 \%$ which is significantly below the FNR of SLNB in patients undergoing upfront surgery for $\mathrm{cN} 0$ breast cancer outside the NST setting. This finding has raised the question whether the SLNB can be safely omitted in this selected group of patients ( $\mathrm{cN} 0$ triple-negative or HER2 positive breast cancer) who achieve complete clinical response (cCR) in the breast as determined by MRI. This is the basis of a new clinical trial that has just commenced recruitment (23).

\section{Clinically Node-Positive Breast Cancer (cN1-2) and NST}

In a previous study, we demonstrated that SLNB alone in patients with initial biopsy proven lymph node involvement undergoing neoadjuvant chemotherapy had a FNR of $13 \%$, which is above the gold standard target of $10 \%$ (24). However, the FNR was reduced if a minimum of three lymph nodes were harvested including the marked biopsyproven lymph node, in addition to the use of immunohistochemistry (IHC) and the dual tracer technique in sentinel node mapping (24).

More recently, we have conducted a pooled analysis of published studies which has shown that harvesting the biopsy proven lymph node that is marked prior to NST is associated with an acceptably low FNR of $6.2 \%$ with a successful retrieval rate of $90 \%(25)$.

There are currently no RCTs confirming oncological safety of omitting ALND for ypN0 disease following NST in patients presenting initially with cN1. However, a recent large European study demonstrated that there was no difference in OS or DFS between patients presenting with cN1-2 disease who were rendered SLNB negative and patients presenting with cN0. The rate of axillary recurrence was reported to be $1.8 \%$ in the former and $1.6 \%$ in the latter (26). In this study that included 688 patients, ALND was not performed when the postNST SLNB was negative. However, some patients received radiation therapy. Furthermore, we have estimated that in the worst-case scenario the probability of compromising OS would be in the region of 1 in 4,000 for a FNR of 5\% in this setting (27).

\section{Targeted Axillary Dissection (TAD)}

Targeted axillary dissection (TAD) refers to the combination of SLNB and marked lymph node biopsy (MLNB), in which lymph nodes are identified and marked radiologically prior the operation and excised during surgery. This has been shown to result in a FNR of $5.2 \%$ according to our recent pooled analysis (25). It is, however, worth highlighting that the degree of overlap between the SLNB and MLNB is approximately $75 \%$ (26). If histological examination of TAD reveals no evidence of residual disease, then a complete ALND and its associated morbidities could be avoided, thus enhancing quality of life. However, if residual disease is identified in the TAD specimen, then escalation of the treatment in the form of surgery (ALND) or radiation therapy is indicated. This approach of treatment escalation should be considered even if the residual disease is minimal, such as micrometastases or isolated tumour cells (ITCs), for which ALND is not indicated in patients who did not receive NST (27).

This escalation of axillary treatment is important in view of the fact that increased residual disease burden has been associated with worsening overall survival with the 5-year survival being reported as $88.9 \%$ for ypN0 compared with $77.6 \%$ for ypN1 and $82 \%$ for ypN0 (i+) and $79.5 \%$ for ypN1 (mi) N2 (28).

TAD is traditionally performed by deploying a marker clip within the biopsy proven lymph node at the time of diagnosis and subsequent localisation by inserting a guidewire under ultrasound control on the day of surgery. In this context, the hydrogel marker (HydroMark) seems to be the best marker in view of the excellent visibility on ultrasonography and sufficiently long half-life (25).

Wire-free techniques have recently been introduced whereby the marker that can be localised by an external detection system is deployed at the

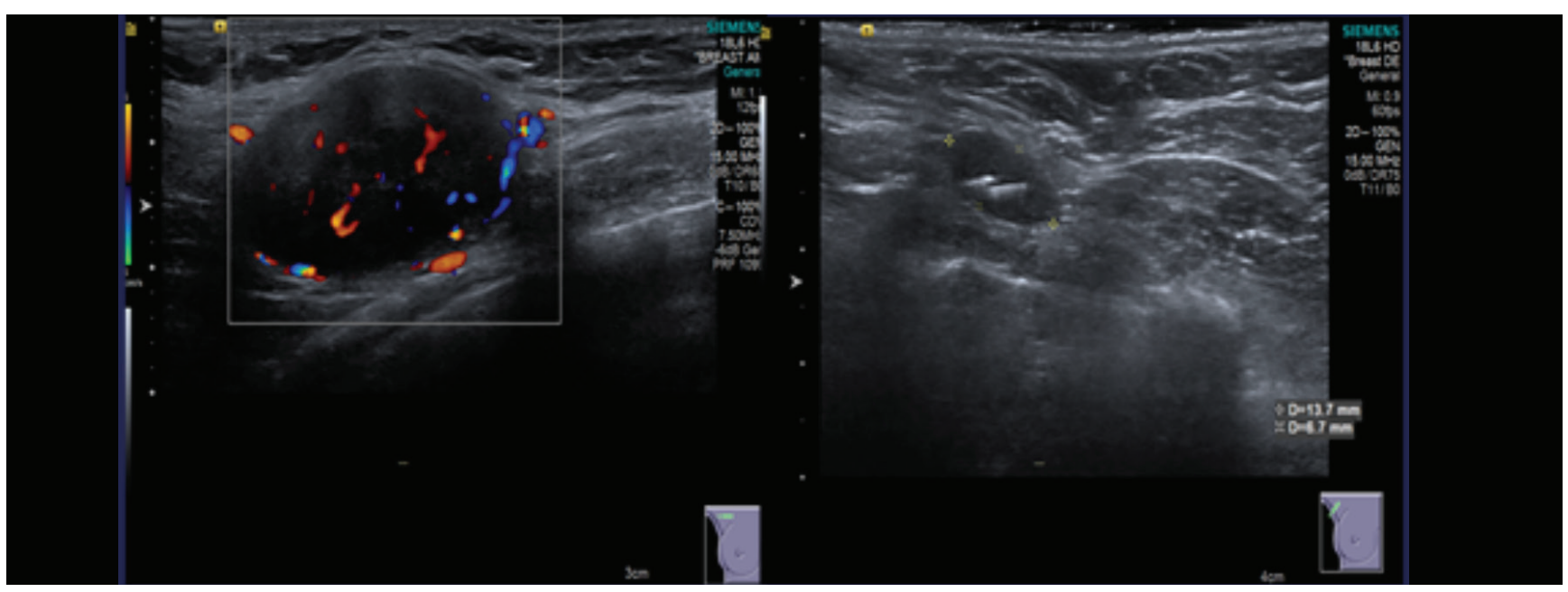

Figure 2. US scan of the right axilla demonstrating a pathological lymph node (cTON1M0 TNBC) with an increased blood flow (left: before NST) that has responded well to NST (right: after NST). The Savi Scout reflector to facilitate targeted axillary dissection (TAD) is seen within the lymph node (right) 
time of lymph node biopsy, so that a second localisation procedure can be avoided (25). These techniques that allow decoupling of radiology and surgery schedules include the use of radioactive iodine seeds, ferromagnetic seeds (MagSeed; Endomag, London, United Kingdom), radio frequency identification tag (LOCalizer, Hologic Inc., Santa Carla, CA, USA) and infrared reflector combined with radar (SAVI SCOUT, Merit Medical, Aliso Viejo, CA, USA) (25) (Figure 3).

The use of radioactive iodine seeds, also known as MARI technique (Marking the Axillary lymph node with Radioactive Iodine seeds), has been curtailed by the extensive regulatory and administrative requirements due to radiation handling. Unlike magnetic seeds and radiofrequency tags, the SAVI SCOUT system generates minimal MRI-void signals and therefore it can be deployed at the time of biopsy in both the primary tumour and the biopsy-proven lymph node (29).

The optimal management strategy of the axilla in patients with cN1 who achieve a cCR in the axilla (ycN0) is the focus of ongoing AXSANA trial (30). This prospective study is not randomised and includes oncological outcome in its primary endpoints.

\section{Can Breast Lumpectomy Be Safely Omitted?}

In patients with occult breast cancer, radiation therapy to the breast has been demonstrated to achieve an OS similar to total mastectomy (31), implying that omission of surgical excision of the occult primary tumour in the breast may not have a detrimental oncological impact. Surgical resection of the primary tumour was not mandatory in some of the trials that compared neoadjuvant and adjuvant chemotherapy. When considering the trials which allowed the omission of primary breast cancer surgery, there is evidence that the distant disease-free survival and OS were not compromised when primary breast cancer surgery is omitted. However, there was a higher incidence of local recurrence (32). A similar observation was reported in a study by Ring
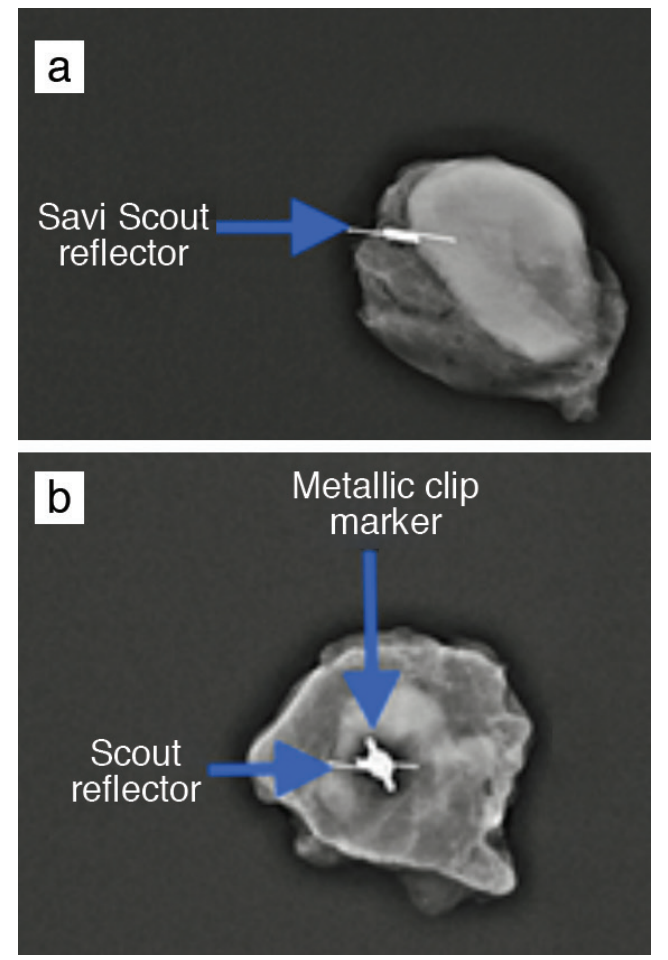

Figure 3. TAD guided by SAVI SCOUT

TAD: Targeted axillary dissection et al. (33) where the omission of lumpectomy after NST was associated with a higher risk of local recurrence without a compromise of OS. However, when the ultrasound response was taken into consideration, the local recurrence rate declined from $33 \%$ to $8 \%$ at five years. These observations raised the possibility of eliminating breast lumpectomy after NST. In order to achieve the optimal outcome, it is logical to consider this form of de-escalation in patients who are most likely to be excellent responders (33).

In this context the molecular subtype plays an important role in patient selection with patients diagnosed with TNBC or HER2 positive disease representing the best candidates. Furthermore, complete radiological response of the primary tumour, as assessed by MRI, would be another important factor to consider in patient selection. For this approach to be effective we should be able to reliably verify pCR without surgery. A meta-analysis of studies evaluating the accuracy of imaging-guided core-biopsy has demonstrated a FNR of $28 \%$ which is significantly above the acceptable target of $10 \%$. However, specificity was as expected to be high at $99 \%$ (34). When considering patients with triple-negative or HER2 positive breast cancer who achieve a complete or partial response on breast imaging the accuracy improves to $98 \%$ with a FNR of $5 \%$ with the use of vacuum assisted core biopsy (9-gauge) obtaining 12 cores in addition to fine needle aspiration cytology (35).

These encouraging results have inspired the NCT 02945579 trial at MD Anderson which has completed accrual. In addition to assessing DFS and OS, the trial included quality of life and cost-effectiveness as part of its primary end points. The use of biomarkers of response, such tumour infiltrating lymphocytes (TILs) (36) and circulating tumour DNA (ctDNA), should be included in future studies as a strategy of monitoring response to treatment and predicting pCR. There is a growing body of evidence that ctDNA reflects residual disease burden and disappearance of this marker in the peripheral blood during NST correlates with pCR and an excellent prognosis (37).

\section{De-Escalation of NST}

Achieving pCR following NST is associated with significantly better DFS and OS, particularly for triple-negative and HER2+ breast cancer (38). Adjuvant chemotherapy in patients who achieved pCR does not seem to improve outcomes (39). This raises the possibility of deescalating NST especially in patients with ER-HER2+ breast cancer who are very likely to attain pCR with pertuzumab, trastuzumab, paclitaxel, and carboplatin, thus avoiding the more toxic anthracyclines (40). Patients with cN0-2 disease who achieve cCR, as determined by MRI and ultrasound, can be selected for surgery after a shorter course of NST to verify pCR and avoid the anthracycline phase of treatment (Figure 4).

\section{Conclusion}

We know that a certain proportion of, but not all, patients with cCR after NST has pCR and pN0. Patients with triple-negative or HER2 positive breast cancer who achieve a complete clinical response after NST, as determined by breast MRI and/or ultrasound, represent excellent candidates for de-escalation of breast cancer surgery. Patients with the cN1-2 disease before NST and had cN0 after NST can undergo TAD as an alternative to complete ALND if pCR is achieved. TAD has been recently facilitated by the advent of novel wire-free and radiation free technologies. 

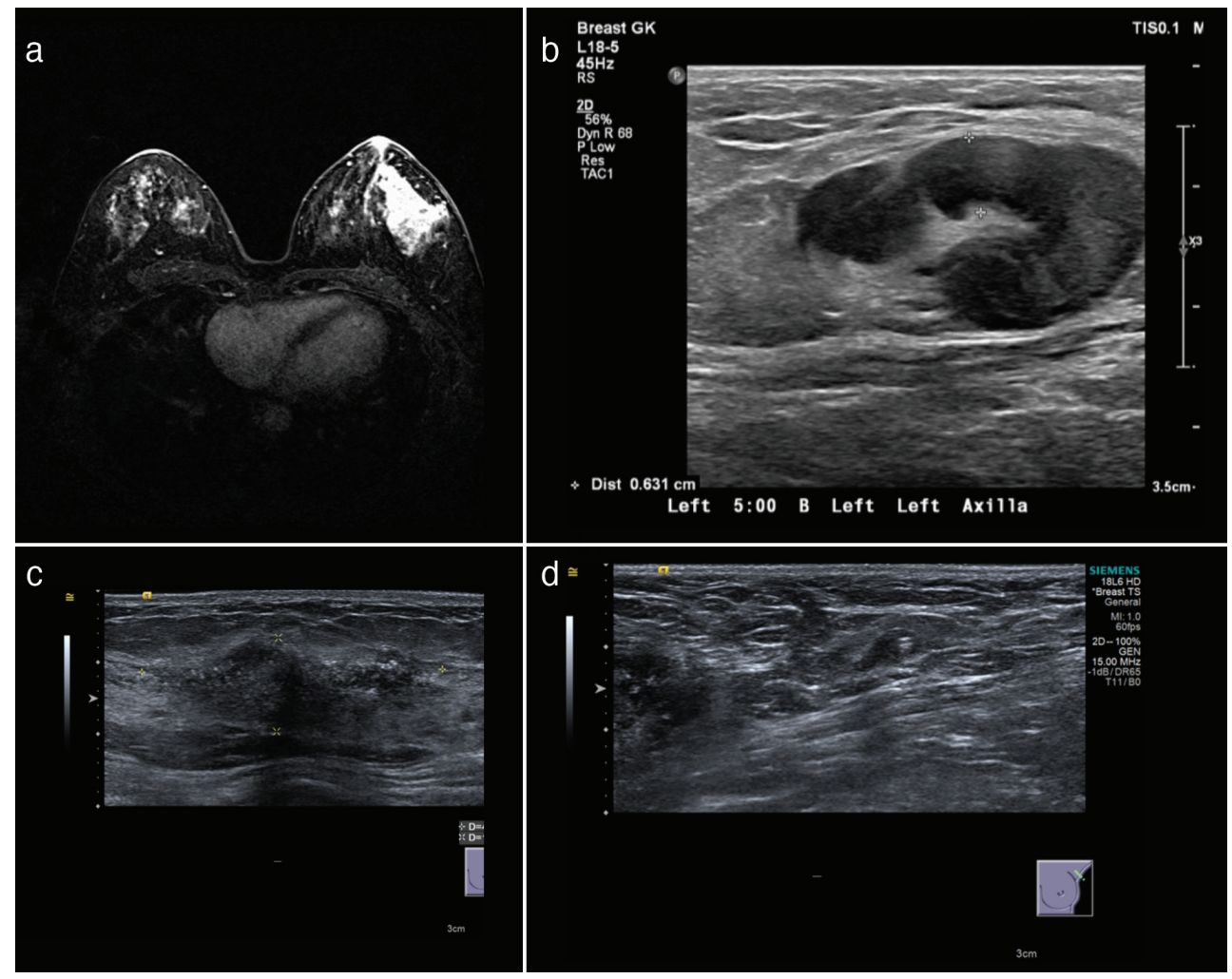

Figure 4. This 45-year-old lady presented with ER-HER2 + T3N1M0 breast cancer. She received 12 weeks of weekly paclitaxel and carboplatin and 3 weekly trastuzumab and pertuzumab followed by surgery that confirmed pCR. (a) MRI prior to treatment demonstrating the large primary tumour; (b) ultrasound scan of the axilla prior to treatment demonstrating the pathological lymph-node; (c) Ultrasound scan of the breast after NST showing non-specific textual change; (d) Ultrasound scan of the left axilla after NST showing a normal giraffe note containing the SAVI SCOUT reflector within it that was subsequently used to guide TAD

HER2: Human epidermal growth factor receptor 2, MRI: Magnetic resonance imaging, NST: Neoadjuvant systemic therapy, TAD: Targeted axillary dissection

The elimination of lumpectomy following an excellent response to NST remains the subject of ongoing clinical trials and is likely to become the new standard of care in selected patients in the future.

Informed Consent: Patients provided consent for anonymised specimen radiographs to be used.

Peer-review: Externally peer-reviewed.

\section{Authorship Contributions}

Surgical and Medical Practices: U.W., K.M.; Concept: K.M.; Design: U.W., K.M.; Analysis and/or Interpretation: U.W., K.M.; Literature Searching: U.W.; Writing: U.W., K.M.

Conflict of Interest: K.M. has received honoraria for providing academic and clinical advisory to Merit Medical. U.W. declared no conflict of interest.

Financial Disclosure: The Breast Cancer Hope Foundation (UK) has provided an educational grant supporting the publication.

\section{References}

1. Mougalian SS, Soulos PR, Killelea BK, Lannin DR, Abu-Khalaf MM, DiGiovanna MP, et al. Use of neoadjuvant chemotherapy for patients with stage I to III breast cancer in the United States. Cancer 2015; 121: 2544-2552.(PMID: 25902916) [Crossref]

2. von Minckwitz G, Huang CS, Mano MS, Loibl S, Mamounas EP, Untch
M, et al. Trastuzumab Emtansine for Residual Invasive HER2-Positive
Breast Cancer. N Engl J Med 2019; 380: 617-628.(PMID: 30516102) [Crossref]

3. Masuda N, Lee SJ, Ohtani S, Im YH, Lee ES, Yokota I, et al. Adjuvant Capecitabine for Breast Cancer after Preoperative Chemotherapy. N Engl J Med 2017; 376: 2147-2159.(PMID: 28564564) [Crossref]

4. Filho OM, Stover DG, Asad S, Ansell PJ, Watson M, Loibl S, et al. Association of immunophenotype with pathologic complete response to neoadjuvant chemotherapy for triple-negative breast cancer: a secondary analysis of the brightness phase 3 randomized clinical trial. JAMA Oncol 2021; 7: 603-608.(PMID: 33599688) [Crossref]

5. Piccart M, Procter M, Fumagalli D, de Azambuja E, Clark E, Ewer MS, et al. Adjuvant pertuzumab and trastuzumab in early her2-positive breast cancer in the aphinity trial: 6 years' follow-up. J Clin Oncol 2021; 39(: 1448-1457.(PMID: 33539215) [Crossref]

6. Fountzila E, Ignatiadis M. Neoadjuvant immunotherapy in breast cancer: a paradigm shift? Ecancermedicalscience 2020; 14: 1147.(PMID: 33574892) [Crossref]

7. Giuliano AE, Ballman KV, McCall L, Beitsch PD, Brennan MB, Kelemen PR, et al. Effect of axillary dissection vs no axillary dissection on 10-year overall survival among women with invasive breast cancer and sentinel node metastasis: the acosog z0011 (alliance) randomized clinical trial. JAMA 2017; 318: 918-926.(PMID: 28898379) [Crossref]

8. Donker M, van Tienhoven G, Straver ME, Meijnen P, van de Velde CJ, Mansel RE, et al. Radiotherapy or surgery of the axilla after a positive sentinel node in breast cancer (EORTC 10981-22023 AMAROS): a randomised, multicentre, open-label, phase 3 non-inferiority trial. Lancet Oncol 2014; 15: 1303-1310.(PMID: 25439688) [Crossref] 
9. Gera R, Kasem A, Mokbel K. Can complete axillary node dissection be safely omitted in patients with early breast cancer when the sentinel node biopsy is positive for malignancy? an update for clinical practice. In Vivo 2018; 32 :1301-1307.(PMID: 30348682) [Crossref]

10. Cortazar P, Zhang L, Untch M, Mehta K, Costantino JP, Wolmark N, et al. Pathological complete response and long-term clinical benefit in breast cancer: the CTNeoBC pooled analysis. Lancet. 2014; 384: 164172.(PMID: 24529560) [Crossref]

11. Montagna G, Tong Y, Ritter M, Levi J, Weber WP, Chen X, et al. Predictors of nodal pathological complete response in asian women with stage ii-iii node-positive breast cancer. Oncology 2021; 99: 359-364. (PMID: 33735903) [Crossref]

12. Kasem J, Wazir U, Mokbel K. Sensitivity, specificity and the diagnostic accuracy of PET/CT for axillary staging in patients with stage i-iii cancer: a systematic review of the literature. In Vivo 2021; 35: 23-30.(PMID: 33402446) [Crossref]

13. Samiei S, de Mooij CM, Lobbes MBI, Keymeulen K, van Nijnatten TJA, Smidt ML. Diagnostic performance of noninvasive imaging for assessment of axillary response after neoadjuvant systemic therapy in clinically nodepositive breast cancer: a systematic review and meta-analysis. Ann Surg 2021; 273: 694-700.(PMID: 33201095) [Crossref]

14. Park S, Yoon JH, Sohn J, Park HS, Moon HJ, Kim MJ, et al. Magnetic resonance imaging after completion of neoadjuvant chemotherapy can accurately discriminate between no residual carcinoma and residual ductal carcinoma in situ in patients with triple-negative breast cancer. PLoS One. 2016; 11: e0149347. doi: 10.1371/journal.pone.0149347. (PMID: 26866475) [Crossref]

15. Expert Panel on Breast Imaging:, Slanetz PJ, Moy L, Baron P, diFlorio RM, Green ED, Heller SL, et al. ACR Appropriateness criteria((R)) monitoring response to neoadjuvant systemic therapy for breast cancer. J Am Coll Radiol 2017; 14(Suppl 11): S462-S75.(PMID: 29101985) [Crossref]

16. Cheng Q, Huang J, Liang J, Ma M, Ye K, Shi C, et al. The diagnostic performance of dce-mri in evaluating the pathological response to neoadjuvant chemotherapy in breast cancer: a meta-analysis. Front Oncol 2020; 10: 93.(PMID: 32117747) [Crossref]

17. Geng C, Chen X, Pan X, Li J. The feasibility and accuracy of sentinel lymph node biopsy in initially clinically node-negative breast cancer after neoadjuvant chemotherapy: a systematic review and meta-analysis. PLoS One 2016; 11: e0162605. doi: 10.1371/journal.pone.0162605. (PMID: 27606623) [Crossref]

18. Hunt KK, Yi M, Mittendorf EA, Guerrero C, Babiera GV, Bedrosian I, et al. Sentinel lymph node surgery after neoadjuvant chemotherapy is accurate and reduces the need for axillary dissection in breast cancer patients. Ann Surg 2009; 250: 558-566.(PMID: 19730235) [Crossref]

19. Classe JM, Loaec C, Gimbergues P, Alran S, de Lara CT, Dupre PF, et al. Sentinel lymph node biopsy without axillary lymphadenectomy after neoadjuvant chemotherapy is accurate and safe for selected patients: the GANEA 2 study. Breast Cancer Res Treat 2019; 173: 343-352.(PMID: 30343457) [Crossref]

20. Burstein HJ, Lacchetti C, Anderson H, Buchholz TA, Davidson NE, Gelmon KE, et al. Adjuvant endocrine therapy for women with hormone receptor-positive breast cancer: American Society of Clinical Oncology Clinical Practice Guideline update on ovarian suppression. J Clin Oncol 2016; 34: 1689-1701.(PMID: 26884586) [Crossref]

21. Riedel F, Heil J, Golatta M, Schaefgen B, Hug S, Schott S, et al. Changes of breast and axillary surgery patterns in patients with primary breast cancer during the past decade. Arch Gynecol Obstet 2019; 299: 10431053. (PMID: 30478667) [Crossref]

22. Barron AU, Hoskin TL, Day CN, Hwang ES, Kuerer HM, Boughey JC. Association of low nodal positivity rate among patients with ERBB2positive or triple-negative breast cancer and breast pathologic complete response to neoadjuvant chemotherapy. JAMA Surg 2018; 153: 11201126. (PMID: 30193375) [Crossref]

23. Reimer T, Glass A, Botteri E, Loibl S, Gentilini OD. Avoiding axillary sentinel lymph node biopsy after neoadjuvant systemic therapy in breast cancer: rationale for the prospective, multicentric EUBREAST-01 trial. Cancers (Basel). 2020;12:3698.(PMID: 33317077) [Crossref]

24. El Hage Chehade H, Headon H, El Tokhy O, Heeney J, Kasem A, Mokbel $\mathrm{K}$. Is sentinel lymph node biopsy a viable alternative to complete axillary dissection following neoadjuvant chemotherapy in women with nodepositive breast cancer at diagnosis? An updated meta-analysis involving 3,398 patients. Am J Surg 2016; 212: 969-981. (PMID: 27671032) [Crossref]

25. Swarnkar PK, Tayeh S, Michell MJ, Mokbel K. The evolving role of marked lymph node biopsy (MLNB) and targeted axillary dissection (TAD) after neoadjuvant chemotherapy (nact) for node-positive breast cancer: systematic review and pooled analysis. Cancers (Basel). 2021; 13: 1539. (PMID: 33810544) [Crossref]

26. Boughey JC, Ballman KV, Le-Petross HT, McCall LM, Mittendorf EA, Ahrendt GM, et al. Identification and resection of clipped node decreases the false-negative rate of sentinel lymph node surgery in patients presenting with node-positive breast cancer (T0-T4, N1-N2) who receive neoadjuvant chemotherapy: results from ACOSOG Z1071 (Alliance). Ann Surg 2016; 263: 802-807.(PMID: 26649589) [Crossref]

27. Patani N, Mokbel K. Clinical significance of sentinel lymph node isolated tumour cells in breast cancer. Breast Cancer Res Treat 2011; 127: 325334. (PMID: 21455668) [Crossref]

28. Wong SM, Almana N, Choi J, Hu J, Gagnon H, Natsuhara K, et al. Prognostic significance of residual axillary nodal micrometastases and isolated tumor cells after neoadjuvant chemotherapy for breast cancer. Ann Surg Oncol 2019; 26: 3502-3509.(PMID: 31228134) [Crossref]

29. Tayeh S, Muktar S, Heeney J, Michell MJ, Perry N, Suaris T, et al. Reflector-guided localization of non-palpable breast lesions: the first reported european evaluation of the SAVI SCOUT(R) system. Anticancer Res 2020; 40: 3915-3924. (PMID: 32620632) [Crossref]

30. Banys-Paluchowski M, Gasparri ML, de Boniface J, Gentilini O, Stickeler E, Hartmann S, et al. Surgical management of the axilla in clinically node-positive breast cancer patients converting to clinical node negativity through neoadjuvant chemotherapy: current status, knowledge gaps, and rationale for the EUBREAST-03 AXSANA study. Cancers (Basel) 2021; 13: 1565. (PMID: 33805367) [Crossref]

31. Tsai C, Zhao B, Chan T, Blair SL. Treatment for occult breast cancer: A propensity score analysis of the National Cancer Database. Am J Surg 2020; 220: 153-160.(PMID: 31753317) [Crossref]

32. Early Breast Cancer Trialists' Collaborative Group (EBCTCG). Longterm outcomes for neoadjuvant versus adjuvant chemotherapy in early breast cancer: meta-analysis of individual patient data from ten randomised trials. Lancet Oncol 2018; 19: 27-39.(PMID: 29242041) [Crossref]

33. Ring A, Webb A, Ashley S, Allum WH, Ebbs S, Gui G, et al. Is surgery necessary after complete clinical remission following neoadjuvant chemotherapy for early breast cancer? J Clin Oncol 2003; 21: 4540-4545. (PMID: 14673041) [Crossref]

34. Lee J. 20 years of The Lancet Oncology: how scientific should oncology be? Lancet Oncol 2020; 21: e461. doi: 10.1016/S1470-2045(20)304228. (PMID: 32888476) [Crossref]

35. Kuerer HM, Rauch GM, Krishnamurthy S, Adrada BE, Caudle AS, DeSnyder SM, et al. A clinical feasibility trial for identification of exceptional responders in whom breast cancer surgery can be eliminated following neoadjuvant systemic therapy. Ann Surg 2018; 267: 946-951. (PMID: 28549010) [Crossref]

36. Kochi M, Iwamoto T, Niikura N, Bianchini G, Masuda S, Mizoo T, et al. Tumour-infiltrating lymphocytes (TILs)-related genomic signature 
predicts chemotherapy response in breast cancer. Breast Cancer Res Treat 2018; 167: 39-47.(PMID: 28905250) [Crossref]

37. Magbanua MJM, Swigart LB, Wu HT, Hirst GL, Yau C, Wolf DM, et al. Circulating tumor DNA in neoadjuvant-treated breast cancer reflects response and survival. Ann Oncol 2021; 32: 229-239.(PMID: 33232761) [Crossref]

38. Spring LM, Fell G, Arfe A, Sharma C, Greenup R, Reynolds KL, et al. Pathologic complete response after neoadjuvant chemotherapy and impact on breast cancer recurrence and survival: a comprehensive meta- analysis. Clin Cancer Res 2020; 26: 2838-2848. (PMID: 32046998) [Crossref]

39. Mandish SF, Gaskins JT, Yusuf MB, Amer YM, Eldredge-Hindy H. The effect of omission of adjuvant radiotherapy after neoadjuvant chemotherapy and breast conserving surgery with a pathologic complete response. Acta Oncol 2020; 59: 1210-1217.(PMID: 32716227) [Crossref]

40. Loi S. Fine-tuning chemotherapy in the era of dual HER2 targeting. Lancet Oncol 2018; 19: 1551-1554.(PMID: 30413380) [Crossref] 